\title{
Dimensional Crossover in a Charge Density Wave Material Probed by Angle-Resolved Photoemission Spectroscopy
}

\author{
C. W. Nicholson, ${ }^{1, *}$ C. Berthod, ${ }^{2}$ M. Puppin, ${ }^{1}$ H. Berger, ${ }^{3}$ M. Wolf, ${ }^{1}$ M. Hoesch, ${ }^{4}$ and C. Monney ${ }^{5}$ \\ ${ }^{1}$ Department of Physical Chemistry, Fritz-Haber-Institut of the Max Planck Society, Faradayweg 4-6, Berlin 14915, Germany \\ ${ }^{2}$ Department of Quantum Matter Physics, University of Geneva, 24 quai Ernest-Ansermet, 1211 Geneva, Switzerland \\ ${ }^{3}$ Institut de la Matière Complexe, École Polytechnique Fédérale de Lausanne, 1015 Lausanne, Switzerland \\ ${ }^{4}$ Diamond Light Source, Harwell Campus, Didcot OX11 ODE Oxfordshire, United Kingdom \\ ${ }^{5}$ Institute of Physics, University of Zurich, Winterthurerstrasse 190, 8057 Zurich, Switzerland
}

(Received 14 October 2016; published 18 May 2017)

\begin{abstract}
High-resolution angle-resolved photoemission spectroscopy data reveal evidence of a crossover from one-dimensional (1D) to three-dimensional (3D) behavior in the prototypical charge density wave (CDW) material $\mathrm{NbSe}_{3}$. In the low-temperature 3D regime, gaps in the electronic structure are observed due to two incommensurate CDWs, in agreement with x-ray diffraction and electronic-structure calculations. At higher temperatures we observe a spectral weight depletion that approaches the power-law behavior expected in one dimension. From the warping of the quasi-1D Fermi surface at low temperatures, we extract the energy scale of the dimensional crossover. This is corroborated by a detailed analysis of the density of states, which reveals a change in dimensional behavior dependent on binding energy. Our results offer an important insight into the dimensionality of excitations in quasi-1D materials.
\end{abstract}

DOI: 10.1103/PhysRevLett.118.206401

In one spatial dimension, reduced screening and a restricted phase space for scattering heavily impact the electronic properties of materials due to the ensuing strong correlations. As a result, the well-known Fermi liquid (FL) concept breaks down, and may be replaced by the Tomonaga-Luttinger liquid (TLL) [1,2], in which correlation functions display power-law behavior. The fundamental excitations of a TLL are collective bosonic modes carrying only spin or charge, rather than electronlike fermionic quasiparticles [3-5]. Furthermore, long-range ordered phases are not stable in a purely 1D system as a result of quantum and thermal fluctuations [6]; thus, a dimensional crossover should be a prerequisite for a $1 \mathrm{D}$ system to enter an ordered phase, as occurs in a number of quasi-1D materials $[7,8]$. This is distinguished by a crossover energy, $E_{C}$, or temperature above which excitations exhibit 1D character, while low-energy excitations behave as in a FL [9-11]. In photoelectron spectroscopy experiments, spectral weight depletion near the Fermi energy has been interpreted as a characteristic of TLL behavior in a variety of systems $[12,13]$, also at very low temperatures $[14,15]$. In contrast, in quasi-1D systems, power-law correlations are expected to be observed only above the dimensional crossover energy or temperature. To date, the properties of the low-temperature phase, in particular how strong one-dimensional correlations are imprinted on it, remain poorly understood [16].

In this Letter, we report a high-resolution angle-resolved photoemission spectroscopy (ARPES) study of $\mathrm{NbSe}_{3}$ single crystals, including the evolution of the electronic structure over a wide temperature range and a mapping of the Fermi surface. We find evidence of a dimensional crossover from $1 \mathrm{D}$ to $3 \mathrm{D}$ as a function of decreasing energy and temperature. CDW gaps in the electronic structure are observed at low temperatures, occurring at momenta consistent with x-ray data and reproduced by theoretical simulations. Conversely, at high temperatures, a power-law suppression of the spectral function is observed, suggestive of $1 \mathrm{D}$ behavior. From the warping of the Fermi surface measured at low temperature, a crossover energy scale of around $E_{C} \approx 110 \mathrm{meV}(1250 \mathrm{~K})$ is extracted based on a tight-binding model. This is corroborated by an analysis of the density of states that reveals 1D behavior only above $E_{C}$. The data presented here attest an intermediate regime in which the bosonization expected for a purely $1 \mathrm{D}$ dispersion is still partially observed while approaching the FL regime of $3 \mathrm{D}$ coherence.

$\mathrm{NbSe}_{3}$ is an archetypical linear-chain compound, which undergoes CDW transitions at $T_{1}=145$ and $T_{2}=59 \mathrm{~K}$ [17] with incommensurate modulation wave vectors $\boldsymbol{q}_{1}=(0,0.243,0)$ and $\boldsymbol{q}_{2}=(0.5,0.263,0.5)$, respectively, in units of the reciprocal lattice parameters $\left(a^{*}, b^{*}, c^{*}\right)$ $[18,19]$. The occurrence of the CDW has been ascribed to Fermi-surface nesting [20,21]. Despite intense research on $\mathrm{NbSe}_{3}$ (for an overview see Refs. [7,22]), detailed information about the electronic dispersion is limited to only a few studies $[20,21]$ by ARPES. Recent work by scanning tunneling microscopy showed a surface CDW transition temperature higher than that in bulk, and confirmed the higher dimensional nature of this material at low temperatures [23], which had previously been observed in $\mathrm{x}$-ray scattering data [24]. In contrast, intriguing reduced 
dimensional behavior at the surface has also been revealed as signatures of soliton behavior [25].

Single crystals of $\mathrm{NbSe}_{3}$ of typical dimensions $20 \times 500 \mu \mathrm{m}^{2}$ were cleaved in vacuum at a pressure lower than $5 \times 10^{-11}$ mbar. ARPES measurements were carried out at the IO5 beam line of the Diamond Light Source [26] over a temperature range $6.5-260 \mathrm{~K}$ with photon energies $20-40 \mathrm{eV}$ (linear horizontal polarization). The angular and energy resolution were $0.2^{\circ}$ and $10 \mathrm{meV}$, respectively. All results presented here have been reproduced by measurements on multiple samples.

A schematic of the crystal structure of $\mathrm{NbSe}_{3}$ is presented in Fig. 1(a), and comprises three distinct triangular prism chains running parallel to the $b$ axis. The Fermi surface obtained by ARPES in the $b c$ plane is shown in Fig. 1(b) and may be compared with that calculated by density functional theory (DFT) in Fig. 1(c) (calculations as

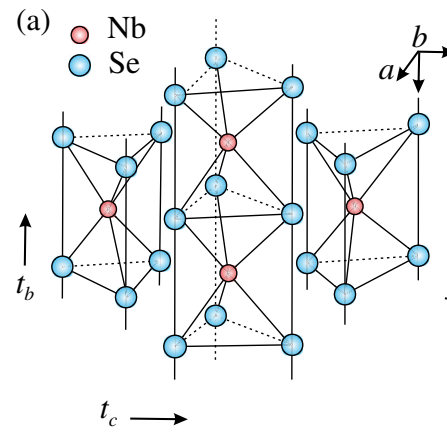

(b)

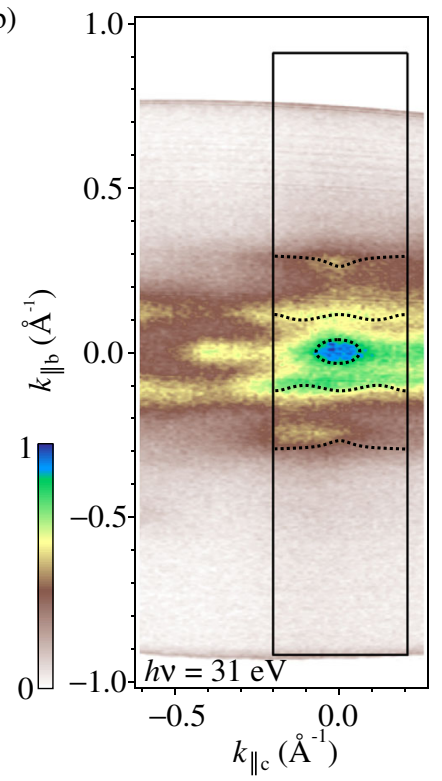

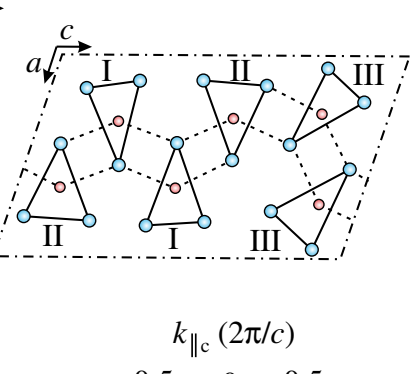

$\begin{array}{lll}-0.5 & 0 & 0.5\end{array}$

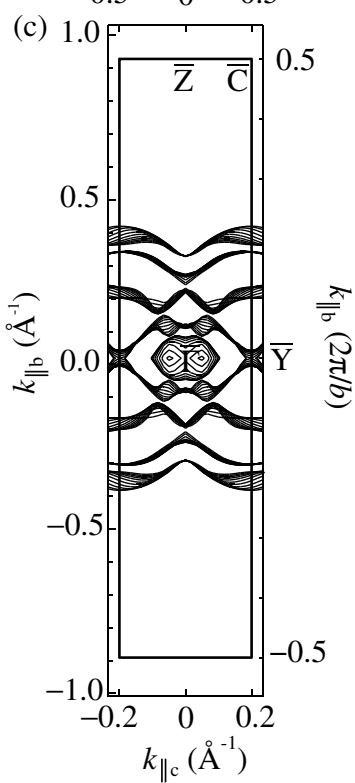

FIG. 1. (a) Schematic crystal structure of $\mathrm{NbSe}_{3}$ (adapted from Ref. [17]). $t_{b}$ and $t_{c}$ are the hopping amplitudes along the chains and along the $c$ axis, respectively, as used in the tight-binding model (see text). (b) Fermi surface obtained at $8 \mathrm{~K}$ with $31 \mathrm{eV}$ photon energy in the $b^{*} c^{*}$ plane. The rectangle shows the first Brillouin zone as in (c). Dotted lines are guides to the eye. (c) DFT Fermi-surface contours in the $b^{*} c^{*}$ plane for various momenta covering the full Brillouin zone along $a^{*}$. in Ref. [20] using Wien2k [27]). While not all five sheets predicted by DFT are resolved at the Fermi level, Fig. 2(b) reveals five bands dispersing up to $E_{F}$ in agreement with the DFT predictions (see Supplemental Material [28] including Ref. [29] for further details, and out of plane dispersion). The dispersion is strongly anisotropic, revealing the quasi-1D nature of the electronic structure. Warping of the Fermi-surface sheets along the $k_{\| c}$ direction (along the $c$ axis in real space) reveals the presence of significant interchain coupling at these low temperatures.

The dispersion of the bands along $k_{\| b}$ at selected $k_{\| c}$ values is given in Figs. 2(a)-2(c). Second derivative plots are presented in Figs. 2(d)-2(f) in order to highlight weak features. A number of features are visible which were not resolved in previous studies $[20,21]$. At $k_{\| c}=0 \AA^{-1}$ we observe three bands dispersing symmetrically around the $\Gamma$ point: the outer band with minimum at $-550 \mathrm{meV}$ and two inner bands with minima at $-260 \mathrm{meV}$. All three bands appear to cross $E_{F}$, although the spectral weight strongly decreases at low binding energies. A very small pocket directly at $E_{F}$ around $\Gamma$ is also observed. At $k_{\| c}=0.2 \AA^{-1}$ three inner bands can be distinguished. At $k_{\| c}=0.35 \AA^{-1}$, in the second Brillouin zone, strong effects of the varying photoemission matrix element lead to different relative intensities of the bands.

Figure 2(d) further reveals a loss of intensity in the outer band at specific energies which appear symmetrically on both sides of $\Gamma$ (arrows). To ensure these are not artifacts of the image processing, we present in Fig. 3(c) energy distribution curves (EDCs) of the raw data at the momenta corresponding to these features. Weak but distinct two-peak structures are observed, centered around $E_{1}=210$ and

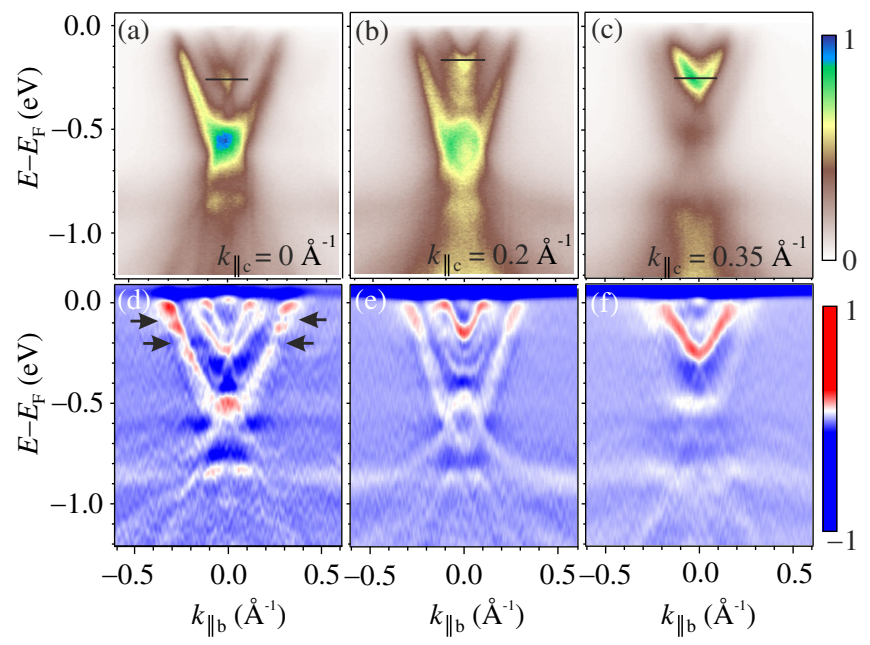

FIG. 2. (a)-(c) Band dispersion along $k_{\| b}$ obtained at $6.5 \mathrm{~K}$ with $31 \mathrm{eV}$ photon energy at the marked $k_{\| c}$ values. Black bars indicate the expected position of the band bottom based on a cosine dispersion along $k_{\| c}$ with bandwidth $4 t_{c}=108 \mathrm{meV}$ (see text). (d)-(f) Corresponding second-derivative plots. The position of the CDW gaps are marked in (d). 

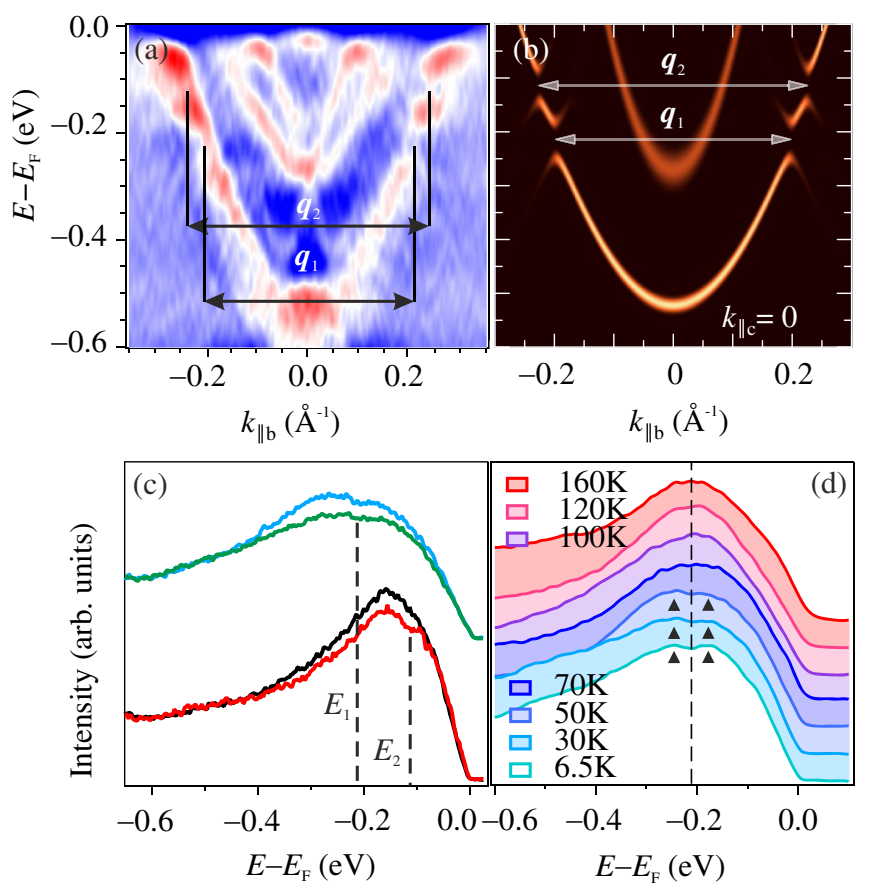

FIG. 3. (a) Enlargement of the data from Fig. 2(d) including the $b^{*}$-axis component of the $\boldsymbol{q}$ vectors for the two incommensurate CDWs: $q_{1, b^{*}}=0.435 ; \boldsymbol{q}_{2, b^{*}}=0.468 \AA^{-1}$ as measured by x-ray diffraction $[18,19]$. (b) Simulation of the spectral function in a CDW system with ordering vectors $q_{1, b^{*}}$ and $q_{2, b^{*}}$ (see text). (c) EDCs of the raw ARPES data at $k_{\| b}=0.21$ (blue), $k_{\| b}=$ -0.20 (green), $k_{\| b}=0.25$ (red), and $k_{\| b}=-0.24 \AA^{-1}$ (black). The center of the CDW gaps is marked by dashed lines. All data are taken at $k_{\| c}=0$ and $T=6.5 \mathrm{~K}$. (d) Temperature dependence of the $\boldsymbol{q}_{1}$ gap at $k_{\| b}=0.21 \AA^{-1}$.

$E_{2}=120 \mathrm{meV}$ below $E_{F}$, which we identify with gaps caused by the $\boldsymbol{q}_{1}$ and $\boldsymbol{q}_{2} \mathrm{CDW}$ superperiodicities, respectively. The scattering vectors deduced from our data, 0.43 and $0.47 \AA^{-1}$, match within errors the $b^{*}$ components of the $\boldsymbol{q}_{1}$ and $\boldsymbol{q}_{2}$ modulation obtained by x-ray diffraction $[18,19]$. This is strong evidence that these gap features occur as a result of the CDWs [30]. In addition, as the temperature is increased, the gap features become weaker up to $50 \mathrm{~K}$ and then disappear, as shown for the $\boldsymbol{q}_{1}$ gap in Fig. 3(d). The fact that the CDW gaps disappear before the bulk transition temperature should not be taken as evidence for a lower CDW transition temperature at the surface, but is more likely due to phonon broadening washing out the signal as $T$ increases. The occurrence of the gap at $210 \mathrm{meV}$ agrees with that observed in Refs. [20,21], although the dispersion that was extracted is not reproduced in our data.

A calculation of the spectral function for a two-band tight-binding model with a two-component CDW is presented in Fig. 3(b) (see Supplemental Material [28] for details including Refs. [31-33]), which can be compared with the corresponding experimental data on Fig. 3(a). The CDW potential opens gaps at momenta connected by the ordering vectors. As is evident from this simulation, the influence of the CDW on the spectral function can be rather weak and the inner band is not affected at all.

We note that the shape of the innermost band in Fig. 2(e) appears to bend away from the Fermi level at $k_{\| b}=0.11 \AA^{-1}$, consistent with previous observations that assigned this behavior to the $\boldsymbol{q}_{2} \mathrm{CDW}$ [21]. We caution that such behavior may result from artifacts due to the secondderivative image processing in the presence of multiple bands and the Fermi edge. The energy and momentum distribution curves presented in the Supplemental Material [28] confirm the absence of a backfolded dispersion. While we cannot rule out gaps at $E_{F}$ that are hidden by the depletion of spectral weight, our data and calculations reveal that the CDW wave vectors $\boldsymbol{q}_{1}$ and $\boldsymbol{q}_{2}$ open gaps only below $E_{F}$, which speaks against a Fermi-surface instability.

We now look in more detail at the Fermi-surface data presented in Fig. 4(a). It is clear that the dispersion in the $\left(k_{\| b}, k_{\| \mathrm{c}}\right)$ plane is strongly anisotropic (i.e., quasi-1D), with a finite warping along $k_{\| c}$ resulting from interchain hopping. Such a quasi-1D dispersion is minimally described by the tight-binding model

$$
E_{k}=-2 t_{b} \cos \left(k_{\| b} b\right)-2 t_{c} \cos \left(k_{\| c} c\right)-\mu,
$$

in which $t_{b}$ and $t_{c} \ll t_{b}$ are the effective hopping amplitudes along the chains and along the $c$ axis, respectively, and $\mu$ is

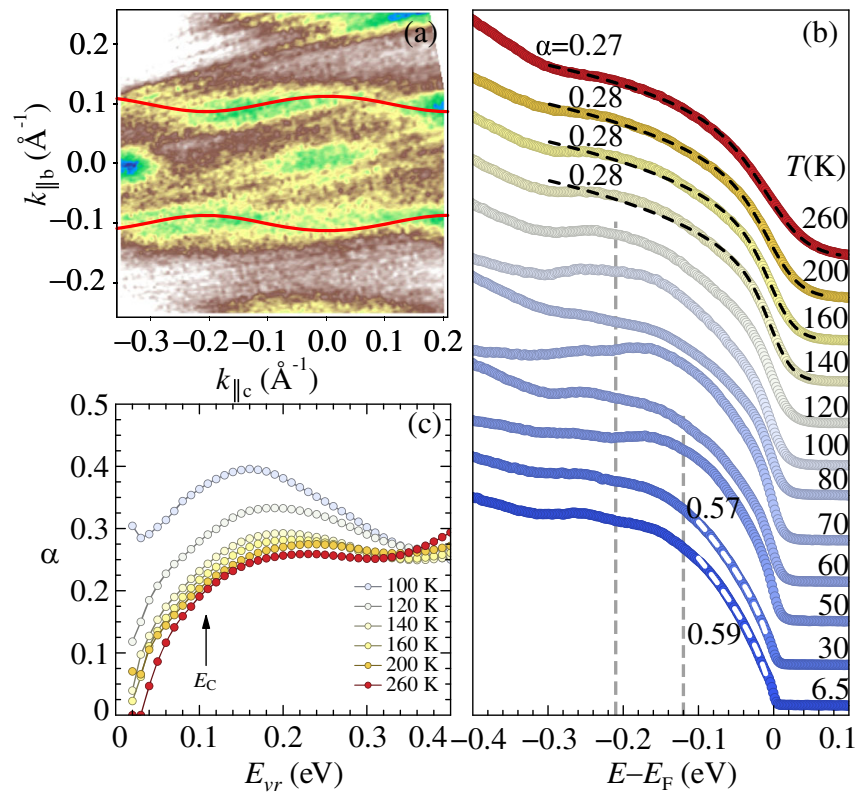

FIG. 4. (a) Fermi surface obtained at $h \nu=22 \mathrm{eV}$, overlaid with the tight-binding model described in the text. (b) $k_{\| b}$-integrated ARPES intensity at $k_{\| c}=0$ for various temperatures. White dashed lines indicate simple power laws with exponents close to 0.6; black dashed lines show best fits to a TLL model spectral function with $\alpha \approx 0.25$; vertical dashed lines indicate the energies of the CDW gaps seen in Fig. 2(d). (c) Energy-dependent exponent showing dimensional crossover at $E_{C}$ for $T>T_{1}$. 
the chemical potential. The transverse bandwidth $4 t_{c} \equiv E_{C}$ defines the energy (temperature) scale at which the system crosses over from 1D to higher dimensional behavior. Excitations with energies $E \gg E_{C}$ are insensitive to the dispersion along $c$ and exhibit 1D character, with the typical power laws expected for a TLL, while excitations with $E<E_{C}$ behave as in a FL. Our Fermi-surface and dispersion data [Figs. 4(a), and Fig. SM2 of the Supplemental Material [28]] in combination with Eq. (1) allow us to extract a value $t_{c}=27 \mathrm{meV}$ (for a derivation see Supplemental Material [28]). This implies a typical energy scale $E_{C}=108 \mathrm{meV}$ above which $1 \mathrm{D}$ signatures should be observed. A simple cross check of this value can be obtained by comparing the relative band bottoms throughout the Brillouin zone along the $k_{\| c}$ direction with that expected from a bandwidth of $4 t_{c}$. These are presented in Figs. 2(a)-2(c) by black horizontal bands and show good agreement.

In Fig. 4(b) we show the $k_{\| b}$-integrated ARPES intensity at $k_{\| c}=0$ within $400 \mathrm{meV}$ of $E_{F}$ and for different temperatures. This quantity approximates the density of states (DOS) multiplied by the Fermi function, apart from inessential corrections associated with the finite experimental resolution and weak $k_{\| c}$ dispersion. We have checked that integrating over smaller $k$ ranges or at other $k_{\| c}$ values does not change the form of the DOS; see the Supplemental Material [28]. At the lowest temperatures, the DOS suppression near $E_{F}$ is markedly different from the expected Fermi edge and resembles a power law. We rule out CDW gaps as a possible explanation for this anomalous suppression of spectral weight: the CDW gaps seen at finite energy in our data have a typical peak-to-peak size of $70 \mathrm{meV}$ [Fig. 3(c)], while the DOS suppression occurs over a much wider energy range. A complete gapping of the Fermi surface would also be inconsistent with the fact that $\mathrm{NbSe}_{3}$ remains metallic even at these low temperatures [17].

The power-law depletion evokes a TLL where the DOS is suppressed like $\left|E-E_{F}\right|^{\alpha}$ at low energy due to the disappearance of single-particle excitations [4]. The nonuniversal exponent $\alpha=\left(K_{\rho}+K_{\rho}^{-1}-2\right) / 4$ (see Supplemental Material [28]) depends on the parameter $K_{\rho}$, which measures the strength of interactions and varies between $0<K_{\rho} \leq 1$. $K_{\rho}=1(\alpha=0)$ corresponds to a noninteracting electron system with flat DOS.

TLL signatures should be searched for at energies and/or temperatures larger than $E_{C}$. Our highest measured temperature $(260 \mathrm{~K})$ is well below the crossover scale $\left(E_{C} \approx 110 \mathrm{meV} \approx 1250 \mathrm{~K}\right)$, such that our whole data set can be regarded as being in a low-temperature regime with respect to the dimensional crossover. In the CDW state, the DOS at $E>E_{C}$ is perturbed by the CDW gaps such that TLL signatures may be masked if present. We, therefore, look for the TLL power law at temperatures higher than $T_{1}=145 \mathrm{~K}$. We fit the data with an expression giving the finite- $T$ DOS of a TLL [34] convolved by our experimental resolution (for further details see Supplemental Material [28]). The fits are performed in a variable energy range $\left[-E_{\mathrm{vr}}, \min \left(E_{\mathrm{vr}}, 4 k_{B} T\right)\right]$ around $E_{F}$ and we extract the exponent $\alpha$ as a function of this range [Fig. 4(c)]. At $E_{\mathrm{vr}}<E_{C}$, the data approach a pure Fermi edge with $\alpha=0$ (3D regime), while at $E_{\mathrm{vr}}>E_{C}$, the fit yields a stable exponent $\alpha \approx 0.25\left(K_{\rho}=0.38\right)$ over a broad energy range (1D regime). The fit includes all data at $E<E_{\mathrm{vr}}$ and, therefore, yields a continuous drop of $\alpha$ towards zero when reducing $E_{\mathrm{vr}}$ below $E_{C}$; this trend is observed at temperatures above $120 \mathrm{~K}$. An analysis of the fit quality (see Supplemental Material [28]) shows that the best fits are obtained for an upper $E_{\mathrm{vr}}$ bound between 0.2 and $0.3 \mathrm{eV}$. Beyond this the DOS upturn from $-0.3 \mathrm{eV}$ due to the band bottom at $-0.6 \mathrm{eV}$ means that the power-law analysis in this range is no longer appropriate. Figure 4(b) shows the best fits with $E_{\mathrm{vr}}=0.3 \mathrm{eV}$ and the corresponding TLL exponents for $T>100 \mathrm{~K}$. For $T<T_{1}$, the fit deteriorates and the extracted exponent becomes strongly energy dependent due to the CDW gaps, while the exponent at low $E_{\mathrm{vr}}$ increases steadily with decreasing $T$ due to the anomalous spectral-weight suppression. The high- $T$ value $K_{\rho}=0.38$ suggests that the interaction has a finite range. Indeed $K_{\rho}>1 / 2$ in the Hubbard model while $K_{\rho}>1 / 8$ in the extended Hubbard model [4]. The value 0.38 thus points to a moderate interaction and locates $\mathrm{NbSe}_{3}$ far from an interaction-driven metal-insulator transition. In contrast, the apparent DOS exponent close to 0.6 at low $T$ [Fig. 4(b)] would indicate much stronger correlations with $K_{\rho}=0.24$. Since our DFT Fermi surface and bands agree with the observed band structure it is unlikely that such a strong renormalization occurs. We conclude that the TLL spectral function is not an appropriate description of the system at these low temperatures where a FL phase is expected. We note that DOS exponents close to 0.6 have been reported for several systems, which may indicate longer-range interactions as in carbon nanotubes [14] and atomic chains $[15,35,36]$ or multiband effects like in lithium purple bronze [37,38].

Our analysis supports the idea that $\mathrm{NbSe}_{3}$ is never observed in a truly TLL regime up to room temperature. Instead a gradual crossover from 1D to 3D occurs, as evidenced by the energy dependence of the $\alpha$ exponent. Such a dimensional crossover is expected in all quasi-1D materials at the energy of the renormalized interchain coupling. This invites one to reconsider previous reports of TLL power-law DOS suppression and check whether the exponents were indeed measured in the 1D regime where the analysis is valid.

In summary, we have performed detailed ARPES measurements over a wide temperature range, which reveal evidence of a dimensional crossover in $\mathrm{NbSe}_{3}$. Such a dimensional crossover is consistent with the quasi-1D warping of the Fermi surface. A careful analysis of the density of states and comparison with expectations for 1D behavior reveal a changing dimensionality of excitations 
above a characteristic energy $E_{C}$. At low temperatures we observe CDW gaps in the electronic structure at the momenta indicated by $\mathrm{x}$-ray diffraction. We expect the analysis presented here to be applicable to other quasi-1D systems due to the generality of finite interchain coupling in real materials, and hope this will stimulate further experimental and theoretical research on the dimensional crossover.

We thank Diamond Light Source for access to beam line I05 (SI10322) and acknowledge useful discussions with Y. Ohtsubo and A.P. Petrović. The work done in Geneva (C. B.) was supported by the Swiss National Science Foundation under Division II. C. M. gratefully acknowledges the support of the SNSF under Grant No. PZ00P2_154867.

*nicholson@fhi-berlin.mpg.de

[1] S. Tomonaga, Prog. Theor. Phys. 5, 544 (1950).

[2] J. M. Luttinger, J. Math. Phys. (N.Y.) 4, 1154 (1963).

[3] J. Voit, J. Phys. Condens. Matter 5, 8305 (1993).

[4] J. Voit, Rep. Prog. Phys. 58, 977 (1994).

[5] T. Giamarchi, Quantum Physics in One Dimension, International Series of Monographs on Physics (Oxford University Press, Oxford, 2003).

[6] N. D. Mermin and H. Wagner, Phys. Rev. Lett. 17, 1133 (1966).

[7] G. Grüner, Rev. Mod. Phys. 60, 1129 (1988).

[8] G. Grüner, Rev. Mod. Phys. 66, 1 (1994).

[9] C. Castellani, C. Di Castro, and W. Metzner, Phys. Rev. Lett. 72, 316 (1994).

[10] E. Arrigoni, Phys. Rev. Lett. 83, 128 (1999).

[11] S. Biermann, A. Georges, A. Lichtenstein, and T. Giamarchi, Phys. Rev. Lett. 87, 276405 (2001).

[12] B. Dardel, D. Malterre, M. Grioni, P. Weibel, Y. Baer, and F. Lévy, Phys. Rev. Lett. 67, 3144 (1991).

[13] G. H. Gweon, S. K. Mo, J. W. Allen, J. He, R. Jin, D. Mandrus, and H. Höchst, Phys. Rev. B 70, 153103 (2004).

[14] H. Ishii, H. Kataura, H. Shiozawa, H. Yoshioka, H. Otsubo, Y. Takayama, T. Miyahara, S. Suzuki, Y. Achiba, M. Nakatake, T. Narimura, M. Higashiguchi, K. Shimada, H. Namatame, and M. Taniguchi, Nature (London) 426, 540 (2003).

[15] Y. Ohtsubo, J.-I. Kishi, K. Hagiwara, P. Le Fèvre, F. Bertran, A. Taleb-Ibrahimi, H. Yamane, S.-I. Ideta, M. Matsunami, K. Tanaka, and S.-I. Kimura, Phys. Rev. Lett. 115, 256404 (2015).

[16] T. Giamarchi, Chem. Rev. 104, 5037 (2004).

[17] N. P. Ong and P. Monceau, Phys. Rev. B 16, 3443 (1977).

[18] R. M. Fleming, D. E. Moncton, and D. B. McWhan, Phys. Rev. B 18, 5560 (1978).
[19] J. L. Hodeau, M. Marezio, C. Roucau, R. Ayroles, A. Meerschaut, J. Rouxel, and P. Monceau, J. Phys. C 11, 4117 (1978).

[20] J. Schäfer, E. Rotenberg, S. D. Kevan, P. Blaha, R. Claessen, and R. E. Thorne, Phys. Rev. Lett. 87, 196403 (2001).

[21] J. Schäfer, M. Sing, R. Claessen, E. Rotenberg, X. J. Zhou, R. E. Thorne, and S. D. Kevan, Phys. Rev. Lett. 91, 066401 (2003).

[22] P. Monceau, Adv. Phys. 61, 325 (2012).

[23] C. Brun, Z.Z. Wang, and P. Monceau, Phys. Rev. B 80, 045423 (2009); C. Brun, Z.-Z. Wang, P. Monceau, and S. Brazovskii, Phys. Rev. Lett. 104, 256403 (2010).

[24] A. H. Moudden, J. D. Axe, P. Monceau, and F. Levy, Phys. Rev. Lett. 65, 223 (1990).

[25] S. Brazovskii, C. Brun, Z.-Z. Wang, and P. Monceau, Phys. Rev. Lett. 108, 096801 (2012).

[26] M. Hoesch, T. K. Kim, P. Dudin, H. Wang, S. Scott, P. Harris, S. Patel, M. Matthews, D. Hawkins, S. G. Alcock, T. Richter, J. J. Mudd, M. Basham, L. Pratt, P. Leicester, E. C. Longhi, A. Tamai, and F. Baumberger, Rev. Sci. Instrum. 88, 013106 (2017).

[27] P. Blaha, K. Schwarz, G. Madsen, D. Kvasnicka, and J. Luitz, An Augmented Plane Wave Plus Local Orbitals Program for Calculating Crystal Properties (Techn. Universität, Wien, Austria, 2001).

[28] See Supplemental Material at http://link.aps.org/ supplemental/10.1103/PhysRevLett.118.206401 for additional data and theoretical models.

[29] A. Damascelli, Phys. Scr. T109, 61 (2004).

[30] The formation of both gaps in the outer band and the observation of both $\boldsymbol{q}_{1}$ and $\boldsymbol{q}_{2}$ modulations on all chains by STM [23] show that Nb orbitals on the three types of chains are coupled and feel both CDW potentials.

[31] L. Covaci, F. M. Peeters, and M. Berciu, Phys. Rev. Lett. 105, 167006 (2010).

[32] A. Weisse, G. Wellein, A. Alvermann, and H. Fehske, Rev. Mod. Phys. 78, 275 (2006).

[33] A. Prodan, N. Ramsak, V. Marinkovic, S. W. Hla, F. W. Boswell, J. C. Bennett, and H. Bohm, Phys. Rev. B 54, 10370 (1996).

[34] K. Schönhammer and V. Meden, J. Electron Spectrosc. Relat. Phenom. 62, 225 (1993).

[35] S. Meyer, L. Dudy, J. Schäfer, C. Blumenstein, P. Höpfner, T. E. Umbach, A. Dollinger, X. Y. Cui, L. Patthey, and R. Claessen, Phys. Rev. B 90, 125409 (2014).

[36] C. Blumenstein, J. Schäfer, S. Mietke, S. Meyer, A. Dollinger, M. Lochner, X. Y. Cui, L. Patthey, R. Matzdorf, and R. Claessen, Nat. Phys. 7, 776 (2011).

[37] F. Wang, J. V. Alvarez, S. K. Mo, J. W. Allen, G. H. Gweon, J. He, R. Jin, D. Mandrus, and H. Höchst, Phys. Rev. Lett. 96, 196403 (2006).

[38] L. Dudy, J. D. Denlinger, J. W. Allen, F. Wang, J. He, D. Hitchcock, A. Sekiyama, and S. Suga, J. Phys. Condens. Matter 25, 014007 (2013). 